Paper presented at the Conference Application of Spectroscopic Techniques on HTSC, CMR and Superlattice Materials held in Kunming, China, August 27-30, 2000.

\title{
SPECTROSCOPIC STUDY OF PARTIALLY-ORDERED SEMICONDUCTOR HETEROJUNCTION UNDER HIGH PRESSURE AND HIGH MAGNETIC FIELD
}

\author{
P.Y. Yu, \\ Department of Physics, University of California, Berkeley \\ and \\ Materials Science Division, Lawrence Berkeley National Laboratory \\ Berkeley, CA 94720, USA \\ G. Martinez and J. Zeman \\ Grenoble High Magnetic Field Laboratory, MPI-FKF/CNRS, \\ Grenoble, FRANCE \\ and \\ K. Uchida \\ Department of Communications and Systems \\ The University of Electro-Communications, Tokyo, JAPAN.
}

\begin{abstract}
Photoluminescence upconversion (PLU) is a phenomenon in which a sample emits photons with energy higher than that of the excitation photon. This effect has been observed in many materials including rare earth ions doped in insulating hosts and semiconductor heterostructures without using high power lasers as the excitation source. Recently this effect has been observed also in partially CuPt-ordered $\mathrm{GaInP}_{2}$ epilayers grown on GaAs substrates. As a spectroscopic technique photoluminescence upconversion is particularly well suited for studying band alignment at heterojunction interface. The value of band-offset has been determined with meV precision using magneto-photoluminescence. Using the fact that the pressure coefficient of electrons in GaAs is higher than those in $\mathrm{GaInP}_{2}$ we have been able to "manipulate" the band-offset at the GaInP/GaAs interface. By converting the band-offset from Type I to Type II we were able to demonstrated that the efficiency of the upconversion process is greatly enhanced by a Type II band-offset.
\end{abstract}


Thin films of random or disordered alloys of $\mathrm{Ga}_{\mathrm{x}} \operatorname{In}_{1-\mathrm{x}} \mathrm{P}$ (where $x \sim 0.5$ ) grown on GaAs (to be abbreviated as $\mathrm{GaInP}$ ) are important materials for visible lasers [Hashimoto91]. They also have potential applications in heterojunction bipolar transistors [Liu92]. For the latter applications it is important to know the band alignment and offset between GaInP and GaAs whose band gap is smaller than GaInP. Using high pressure photoluminescence (PL) measurements the disordered GaInP/GaAs interface was found to be type I with the conduction band offset $\sim 0.1 \mathrm{eV}$ [Chen91]. GaInP grown on GaAs by techniques such as MBE or MOCVD tends to self-organize into a monolayer superlattice along two of the equivalent [111] crystallographic axes [Gomyo87]. This ordering is known as CuPt ordering. The band gap in the ordered samples is reduced by as much as $0.1 \mathrm{eV}$ when compared with the disordered alloy [Kondow88]. It was generally assumed that the band alignment remains type I (for a definition of type I and II band alignments see, for example, [Yu99]). Although Liu et al. [Liu95] have proposed that the band alignment between the partially ordered GalnP and the GaAs substrate is type II, this band assignment was not generally accepted.

Driessen [Driessen95] and Su et al. [Su 96] independently reported that partially ordered GaInP/GaAs samples exhibited photoluminescence upconversion with unusually high efficiency. Driessen [Driessen95] assumed that the band offset between GaAs and GaInP was of type I and suggested that the upconversion process involve the excitation of both electrons and holes via Auger processes occurring near the interface. However, given the low excitation power of the experiment, Su et al. [Su96] concluded that, the probability for exciting both the electron and hole via Auger processes would be too small to explain the observed upconversion efficiency. They suggested instead that electrons probably transferred spontaneously from GaInP to GaAs as a result of a type II band alignment. This idea was not generally accepted also since the band alignment at the GaInP/GaAs junction was believed to be type $I$.

It is not easy to determine the value of the band offset especially if the magnitude of the band offset is very small as assumed by Su et al.. Experimental techniques for measuring band offsets like photoemission has relatively poor energy resolutions with typical uncertainties larger than $20 \mathrm{meV}$ [Waldrop96; Almeida 99]. Other technique such as ballistic-electron-emission-microscopy [O'shea96, O'shea97] cannot probe an interface 
too far below the surface and hence is susceptible to surface effects. One technique which is well suited to measuring a small type II band offset is photoluminescence (PL) at low temperature. In case of a small type II band offset it is often possible to observe recombination of both spatially direct and indirect transitions occurring in the vicinity of the interface. For example, if a small amount of electrons is present in both GaAs and $\mathrm{GaInP}$ at non-zero temperatures at the $\mathrm{GaInP} / \mathrm{GaAs}$ interface then both spatially direct and indirect transition arising from their recombination with holes in GaAs can be observed. Since both transitions involve the same holes in GaAs, their energy difference is a precise measurement of the conduction band offset. The identification of these transitions can be facilitated by applying magnetic field to the sample and measuring the effective masses of the carriers via their cyclotron frequencies. The magnetic field can be complemented by the application of hydrostatic pressure, which can modify the band offset. In this article we shall summarize the various photoluminescence experiments carried out under high magnetic field and/or high pressure to determine the conduction band offset in partially ordered GaInP/GaAs. We note that many of these results have been reported separately in a number of publications. The relevant publications will be cited at the appropriate places in this article.

The GaInP samples studied in this article were all grown on GaAs substrates by low-pressure metal-organic vapor phase epitaxy (LP-MOVPE) and latticematched to GaAs with an accuracy of $\Delta a / a<10^{-3}$ measured by Double Crystal X-ray Diffractometry. These samples can be divided into two series for convenience according to their different growth conditions, structures and types of substrate. The first series of partially ordered GaInP samples have been labeled as $S 1$ to $S 4$ [Uchida 94].They have been grown with a mass-production type vertical LP-MOVPE reactor in Shin-Etsu Handoutai Co., Japan. The growth temperature was $700^{\circ} \mathrm{C}$ with a (group V)/(group III) element ratio and growth rate of 100 and $3 \mu \mathrm{m} /$ hour, respectively. The sample structures are all the same: GaInP $(1 \mu \mathrm{m}) / \mathrm{GaAs}$ buffer layer $(0.1 \mu \mathrm{m}) / \mathrm{GaAs}$ substrate. However, the degree of ordering in the GaInP layers was controlled by cutting the GaAs substrates in such a way that the (100) surface has different degrees of mis-orientation towards the [011] direction. The degrees of mis-orientation are $0^{\circ}, 5^{\circ}, 10^{\circ}$ and $15^{\circ}$ for $\mathrm{S} 1, \mathrm{~S} 2$, S3 and S4, respectively. S1 is most ordered and shows the lowest PL peak energy of 
$1.86 \mathrm{eV}$ at room temperature, while S4 is the least ordered and shows the highest PL peak energy of $1.92 \mathrm{eV}$. The $T$ series consisting of three samples were grown for the study of the PL upconversion by LP-MOVPE in Nippon Sanso Co. Japan, with a single wafer handling type vertical reactor [Su96; Zeman97]. These samples were fabricated on $1 \mathrm{~mm}$ thick (100) GaAs substrates with no mis-orientations. Different growth temperatures were employed to control the degree of ordering, and these samples have been labeled as T650, T700 and T750 according to their growth temperatures which varies from $650{ }^{\circ} \mathrm{C}$ to $750{ }^{\circ} \mathrm{C}$. The thickness of the GaInP layers in these samples is also $1 \mu \mathrm{m}$ and they were grown with a V/III ratio of 200 for the GaInP layer and a ratio of 50 for the GaAs buffer layer. The degree of ordering is found to be highest in T700 while lowest in T750 as indicated by the energy of their low temperature emission peaks (see Table 1). Finally, the expected free carrier concentrations for the GaAs and GaInP layers in all our samples are of the order of $10^{14} \mathrm{~cm}^{-3}$ for holes and $10^{16} \mathrm{~cm}^{-3}$ for electrons, respectively, based on considerations of the experimental V/III ratios during growth.

Table 1. The low temperature PL emission peak energies and band offsets of the three series of partially ordered-GaInP samples studied in this article.

\begin{tabular}{llll}
\hline Sample & $\begin{array}{l}\text { PL peak energy } \\
(\mathrm{eV})\end{array}$ & $\begin{array}{l}\Delta \mathrm{E}_{\mathrm{c}}=\mathrm{E}_{\mathrm{c}}(\mathrm{GaAs})- \\
\mathrm{E}_{\mathrm{c}}(\mathrm{GaInP}) \text { at } 1 \mathrm{bar} \\
(\mathrm{meV})\end{array}$ & $\begin{array}{l}\text { Upconversion at } \\
1 \text { bar }\end{array}$ \\
\hline S1 & 1.906 & 2.4 & No \\
S2 & 1.956 & & No \\
S3 & 1.983 & & No \\
S4 & 1.989 & -1.3 & Yes \\
T650 & 1.895 & -3 & Yes \\
T700 & 1.888 & -3 & Yes \\
T750 & 1.928 & & \\
\hline
\end{tabular}

Most of our experiments involve excitations of emission from samples via laser excitation. The excitation lasers are either visible lasers such as $\mathrm{Ar}^{+}$or $\mathrm{Kr}^{+}$ion laser or a tunable near-IR Ti:sapphire laser. The samples are maintained at low temperature inside a cryostat. The photoluminescence from the sample is analyzed by a Spex double monochromator and detected by photon-counting technique. To vary the pressure on the samples, the sample is lapped to dimension of $<1 \mathrm{~mm}$ and loaded into a diamond anvil cell (DAC). This cell has been designed to allow it to be removed from the hydraulic press used to apply the pressure. The pressure is maintained inside the cell via a lock- 
ring. The sample is placed inside a hole drilled into a metal gasket and surrounded by a pressure media which is liquid nitrogen. The pressure applied to the sample is measured by the standard ruby fluorescence technique. The high magnetic field measurements were performed with the high static magnetic fields (up to 23T) produced by modified Bittertype magnets at the Grenoble High Magnetic Field Laboratory (GHMFL). For experiments under both high pressure and high magnetic field, there are additional severe restrictions on the size of the DAC imposed by the diameter of the bore of the high field magnet. In these experiments we used a small DAC made from copper beryllium whose design has been described in details already in [Zeman96] (see Fig. 1(a)). The cell was fixed at the bottom of a long insert filled with helium exchange gas. The entire assembly was immersed into a helium bath cryostat placed into the bore of the magnet. Radiation for exciting the sample and the signal collected from the sample were all transmitted into or out of the cryostat via optical fibers. In order to minimize the background signal generated in the optical fibers by the excitation laser, we designed a special optical splicing system consisting of a beam-splitter and three optical fibers shown schematically in Fig. 1 (b).Other details of the optical system have been described elsewhere and will not be repeated here[Yu00].

As mention in the introduction the idea that the band alignment at the partially ordered-GaInP/GaAs may be type II and is responsible for the high PL upconversion efficiency in this system was proposed by Su et al. [Su96]. This was not generally accepted although subsequent theoretical calculation of Froyen $e t$ al. [Froyen96] suggests that the band offset can be type II in more ordered GalnP and GaAs interface. As seen from Table 1 there is no strong correlation between upconversion and the degree of order in GaInP as indicated by the band gap reduction. For example, all three $\mathrm{T}$-series samples show upconversion but not sample S1, although the PL peak energy of S1 is smaller than that of sample T750 (see Table 1). To determine whether there is any correlation between the band alignment and upconversion in the $\mathrm{T}$ series we have studied their magnetophotoluminescence or magneto-PL in short. When excited by laser with photon energy above the band gap of GaAs but below the band gap of the GaInP layer, the PL spectra of all three samples in the $\mathrm{T}$-series exhibit a broad structure located around $1.49 \mathrm{eV}$ in addition to exciton peak near the band gap of the GaInP layer as a result of upconversion. 
The intensity of the up-converted PL (to be abbreviated UPL) is strongest in T700 so we shall concentrate on this sample first. Figure 2 shows both the "normal" (above-gap excited) PL and upconverted PL spectra in sample T700.

The structure around $1.49 \mathrm{eV}$ is attributed to the GaAs substrate based on its energy. It may be due to either a spatially indirect transition which would imply that the band offset is type II or deep levels lying within the gap of GaAs. These two possibilities can be distinguished by the effect of high magnetic field on its peak energy.

It is well established that under a magnetic field $B$, the energy of a charged free carrier of mass $m^{*}, \mathrm{~g}$-factor $g^{*}$ and spin $S$ undergoes a shift $\Delta E_{n}$ (see, for example, Chapter 9 of [Yu99]):

$$
\Delta E_{n}=\hbar \omega_{c}(n+1 / 2)+g^{*} \mu_{0} S \cdot B
$$

where

$$
\mu_{0}=\hbar e /\left(2 m_{0} c\right)
$$

is the Bohr magneton and

$$
\omega_{c}=e B /\left(m^{*} c\right)
$$

is the cyclotron frequency and $n$ is an integer $\geq 0$. In Eqs. (1) to (3) $\hbar$ is the Planck's constant, $e$ is the electronic charge, $m_{o}$ is the mass of electron in free space and $c$ is the speed of light (CGS units are used in this article). On the right hand side of Eq. (1), the first term gives rise to the Landau quantization of the cyclotron orbits (and is known as the Landau diamagnetic contribution) while the second term represents the Zeeman splitting $\Delta E_{Z}$. For electrons in the lowest conduction band of GaAs, $g^{*}=-0.44$, $m^{*}=0.067 m_{o}$ and $S=1 / 2$ results in a ratio of $\hbar \omega_{d} / \Delta E_{Z}$ equal to $\sim 16$. Thus for the purpose of this article we expect the Landau term to be dominant. We note that the Landau contribution is linear in $B$ with a slope which depends on the reciprocal of the mass of the carrier and therefore can be used, in principle, to identify the origin of carriers involved in optical transitions.

The magnetic dependent results are shown in Fig. 3(a). Under high magnetic field the emission structure around $1.49 \mathrm{eV}$ from GaAs become resolved clearly into two peaks and both exhibit a linear shift with $B$ but with different slopes. From these slopes 
effective masses of $0.07 m_{0}$ and $0.084 m_{0}$ [Zeman97] have been obtained for the high and low energy peaks, respectively. The mass of $0.07 m_{o}$ is consistent with the electron mass in GaAs and suggests that the higher energy transition involves recombination of free electrons in GaAs with holes presumably bound to carbon acceptors in GaAs. Similarly the mass of the lower energy peak suggests that it involves free electrons in GaInP and bound holes in GaAs and is, therefore, a spatially indirect transition. Thus the crucial result we obtained was that the spatially indirect transition had lower energy than the spatially direct transition suggesting that the band alignment in the sample T700 is type $I I$. From the separation of these PL peaks extrapolated back to zero field a conduction band offset $\Delta E_{c}=E_{c}(\mathrm{GaInP})-E_{c}(\mathrm{GaAs})=-3 \mathrm{meV}$ was obtained. Such a small band offset would have been difficult to determine using another technique. Similar spatially indirect transitions were identified in the other two samples T650 and T750 by magnetophotoluminescencence. However, the band offsets were found to be also in the -2 to -3 meV range as can be seen in Table 1. Thus no significant difference was found between the band offsets among samples in the T-series, in spite of the fact that their PL peak energies are quite different.

For comparison we show also in Fig. 3 (b) the magnetic field dependence of the peaks in the UPL spectra from GalnP in sample T700. Both peaks in this figure shift linearly with $B$ up to $20 \mathrm{~T}$ indicating that they correspond to recombination of either free carriers only or of free carriers with localized particles. The slopes of the magnetic field dependence suggest that the higher energy peak involves the recombination of free holes in GaInP (with the effective mass $=0.24 \mathrm{~m}_{0}$ ) but localized electrons while the lower energy peak corresponds to the recombination of free electrons in GaInP (effective mass $=0.084 m_{0}$ ) with localized holes [Zeman97]. The nature of the traps responsible for localizing carriers in GaInP is not known. However, it is well established that defects associated with stacking faults or anti-phase domain boundaries are very abundant near the GaInP/GaAs interface. It is quite plausible that the abundance of such trapping centers near this interface is partially responsible for the strong upconversion efficiency in this system.

We have pointed out earlier that PL peak emission energy in the sample $\mathrm{S} 1$ is higher than T700 but it does not exhibit any detectable UPL. The lack of UPL suggests 
that S1 may have a type I GaAs/GaInP interface. Furthermore, since the S1 GaInP PL peak energy is not too far from that of T750 the magnitude of its band offset is probably small also. Experimentally it has been determined that the pressure coefficients of the direct band gap at $\Gamma\left(d E_{g} / d P\right)$ in GaAs, disordered-GaInP and partially ordered-GaInP as reported by various groups are: 105, 82 and $65 \mathrm{meV} / \mathrm{GPa}$, respectively [Yu78; Patel89; Chen91; Uchida94; Zeman99]. Since the pressure coefficient in GaAs is much larger than in partially ordered-GalnP, it may be possible to use hydrostatic pressure to convert the band alignment in sample S1 from type I to type II!

The PL spectra of the GaAs substrate in S1 at atmospheric pressure and zero magnetic field $(B=0)$ are shown in Fig. 4.

One notices immediately that under relatively low spectral resolution this spectrum resembles the one from T700 (see Fig. 2) in that there is a structure around 1.49 $\mathrm{eV}$. The only difference is that the sharper peak at higher energy near the band gap of GaAs is relatively stronger in S1 than in T700. The magnetic field dependence of both peaks at $B<7 \mathrm{~T}$ does not exhibit any unusual behavior. The field dependence of the lower energy peak is similar to the well known donor-acceptor transition in GaAs [Zemon86]. The field dependence of the higher energy peak indicates that free electrons and holes in GaAs are involved. Unlike the case of T700 no field induced splitting was observed in the $1.49 \mathrm{eV}$ structure and therefore no peak attributable to spatially indirect transition can be identified. These results suggest that the GaAs/GaInP interface in sample $\mathrm{S} 1$ is indeed type $\mathrm{I}$ at atmospheric pressure. Under a pressure of $>1.2 \mathrm{GPa}$ we find that UPL starts to appear suggesting that pressure has indeed converted the band alignment in S1 from type I to II. In addition to inducing UPL, pressure causes an additional peak to appear in between the donor-acceptor recombination peak and free electron-free hole structures in GaAs.

Next we use the high pressure optical system shown in Fig. 1 to study the magnetic field dependence of the PL spectra from both GaAs and GaInP under high pressure. The low field $(B<7 \mathrm{~T})$ dependence of the $1.49 \mathrm{eV}$ peak suggests that involves a free electron in GaInP with effective mass $=0.086 m_{o}$. This indicates that the new peak is a spatially indirect transition involving free electrons in GaInP and bound holes in GaAs similar to the peak observed in T700 at atmospheric pressure. The dependence of 
the intensity and peak position of this new peak at high field turns out to be quite unusual and will be discussed in more details in the next paragraph. At this point it suffices to note that the conduction band offset $\Delta E_{c}=E_{c}(\mathrm{GaInP})-E_{c}(\mathrm{GaAs})$ in the sample $\mathrm{S} 1$ is found to be pressure dependent and it varies from $-4 \mathrm{meV}$ at $1.4 \mathrm{GPa}$ to $-11 \mathrm{meV}$ at 2.6 GPa. From these results in sample $\mathrm{S} 1$ we determined the pressure dependence of the band offset $\left|d\left(\Delta E_{c}\right) / d P\right|$ at the GaAs/GaInP heterojunction to be $4.6 \pm 0.6 \mathrm{meV} / \mathrm{GPa}$. Using these results we deduce that the band alignment in $\mathrm{S} 1$ at ambient pressure is indeed type I with $\Delta E_{c}=2.4 \mathrm{meV}$. Thus the high pressure magneto-PL results demonstrate conclusively that the interface between $\mathrm{GaAs}$ and partially ordered-GaInP in sample $\mathrm{S} 1$ can be converted from type I to type II with pressure. It is interesting to note that such a small type I band offset is already sufficient to suppress UPL in S1 at atmospheric pressure. Furthermore, efficient UPL becomes observable once the band alignment becomes type II. Our combined experiments in the T-series and $\mathrm{S} 1$ samples show that, at least in the GaAs/GaInP system, a type II band alignment is an important condition for efficient UPL and hence the mechanism responsible for the UPL process observed in this system is most likely the two-step two- photon absorption mechanism proposed by $\mathrm{Su}$ et al. [Su96].

In conclusion, we have utilized high magnetic field to measure the band offset and alignment between partially-ordered GaInP and its substrate GaAs. By combining high pressure with high magnetic field measurements we have demonstrated that a Type II band alignment can enhance the efficiency of photoluminescence upconversion.

Acknowledgments: Research at Berkeley was supported by the DOE through contract DE-AC03-76SF00098, The Grenoble High Magnetic Field Laboratory is Laboratoire conventionné á l'UJF et I'TNPG de Grenoble", JZ acknowledges partial support from the grant ERBCHGECT 930034 of the European Commission, Grant 202/96/0021 from the Grant Agency of the Czech Republic and Grant A1010809 of the Academy of Sciences of the Czech Republic. We also like to acknowledge collaborations with Z.P. Su, K.L. Teo, and S.Julian. 


\section{REFERENCES}

[Almeida 99] J. Almeida, L. Sirigu, G. Margaritondo, P. Da Padova, C. Quaresima and P. Perfetti, J. Phys. D 32, 191 (1999).

[Chen91] J. Chen, J. R. Sites, I. L. Spain, M. J. Hafich and G. Y. Robinson, Appl. Phys. Lett. 58, 744 (1991).

[Driessen95] F.A.J.M. Driessen, Appl. Phys. Lett. 67, 2813 (1995).

[Froyen96] S. Froyen, A. Zunger and A. Mascarenhas, Appl. Phys. Lett. 68, 2852 (1996).

[Gomyo87] A. Gomyo, T. Suzuki, K. Kobayashi, S. Kawata and I. Hino, Appl. Phys. Lett. 50, 673 (1987).

[Hashimoto91] J. L.Hashimoto, T. Katsuyama, J. Shinkai, I. Yoshida and H. Hayashi, J. Appl. Phys. 61, 1713 (1991).

[Kondow88] M. Kondow, H. Kakibayashi, S. Minagawa, Y. Inoue, T. Nishino and Y. Hamakawa, Appl. Phys. Lett. 53, 2053 (1988).

[Liu92] W. Liu and S. K. Fan, IEEE Electron Device Lett. 13, 510 (1992).

[Liu95] Q. Liu, S. Derksen, A. Lindner, F. Scheffer, W. Prost, and F.-J. Tegude, J. Appl. Phys. 77, 1154 (1995).

[O'shea96] J. J. O'shea, C. M. Reaves, S. P. DenBaars, M. A. Chin, and V. Narayanamurti, Appl. Phys. Lett. 69, 3022 (1996).

[O'shea97] J. J. O'shea, E. G. Brazel, M. E. Rubin, S. Bhargava, M. A. Chin, and V. Narayanamurti, Phys. Rev. 56, 2026 (1997).

[Patel89] D. Patel, J. Chen, S. R. Kurtz, J. M. Olson, J. H. Quigley, M. J. Hafich and G. Y. Robinson, Phys. Rev. B 39, 10978 (1989).

[Su96] Z. P. Su, K. L. Teo, P. Y. Yu and K. Uchida, Solid State Commun. 99, 933 (1996).

[Uchida94] K. Uchida, P. Y. Yu, N. Noto, and E. R. Weber, Appl. Phys. Lett. 64, 2858 (1994).

[Uchida97] K. Uchida, T. Arai and K. Matsumoto, J. Appl. Phys. 81, 771 (1997).

[Waldrop96] J. R.Waldrop, and R.W. Grant, Appl. Phys. Lett. 68, 2879 (1996). 
[Yu78] P. Y. Yu and B. Welber, Solid State Commun. 25, 209 (1978).

[Yu99] P. Y. Yu and M. Cardona, Fundamentals of Semiconductors: Physics and Materials Properties, $2^{\text {nd }}$ Edition (Springer-Verlag, Berlin, 1999).

[Yu00] P. Y. Yu, G. Martinez, J. Zeman, K. Uchida, to appear as a chapter in a book on Ordering in Semiconductors, edited by G. Stringfellow and A. Macarenhas, and to be published by Kluwer.

[Zeman96] J. Zeman, G. Martinez, P. Y. Yu, K. Uchida, Proc. ICPS-23, Berlin 1996, eds. M. Scheffler, R. Zimmermann, (World Scientific, Singapore 1996), p. 493.

[Zeman97] J. Zeman, G. Martinez, P.Y. Yu and K. Uchida, Phys. Rev. B 55, R13428(1997).

[Zeman99] J. Zeman, S. Jullian, G. Martinez, P. Y. Yu, K. Uchida, Europhys. Lett. 47, 260, (1999).

[Zemon86] S. Zemon et al., J. Appl. Phys. 59, 2828 (1986). 


\section{FIGURES \& CAPTIONS}

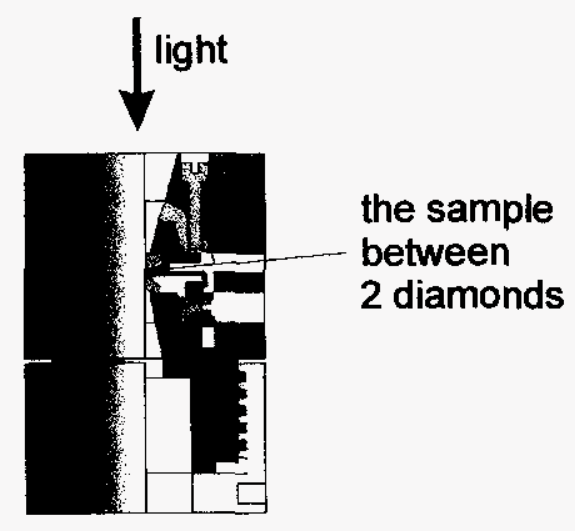

a)

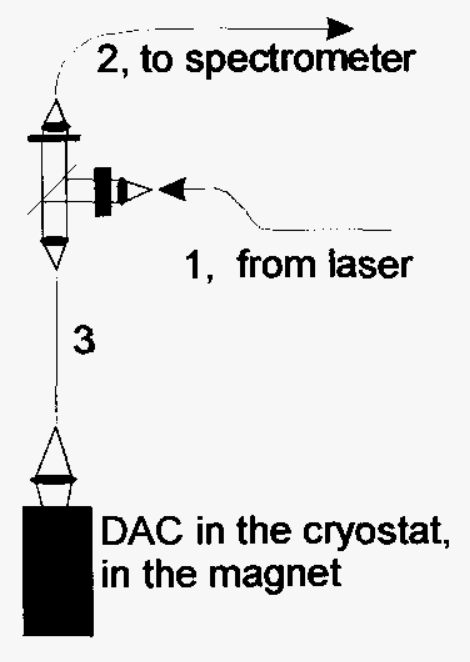

b)

Figure 1. Schematic diagram of the diamond anvil high pressure cell (a) and optical system for performing measurements under high pressure and high magnetic fields (b).

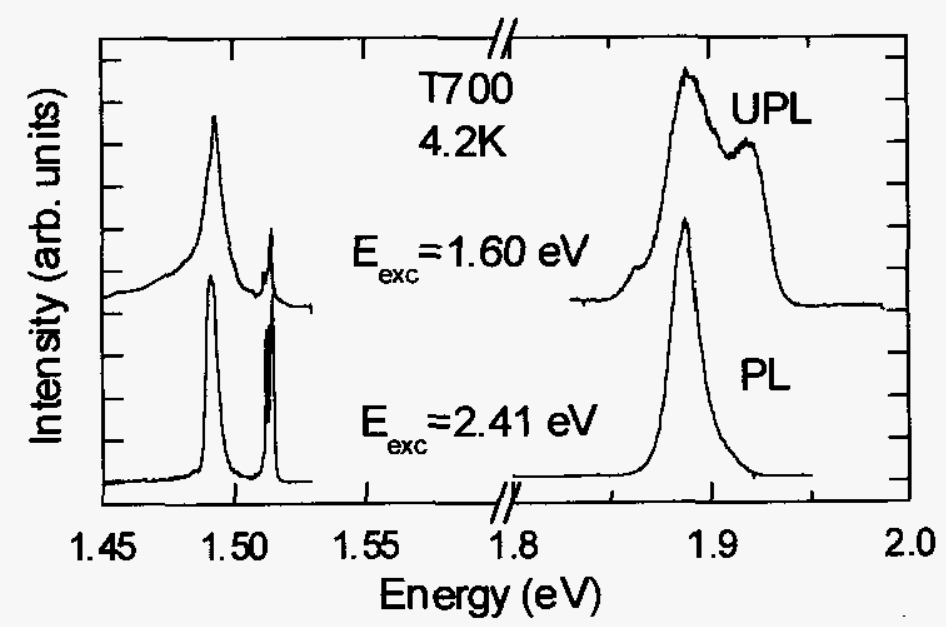

Figure 2. Photoluminescence of sample T700 for two different excitation laser energies showing both the normal and upconverted (labeled, respectively, as PL and UPL) from GaInP. 

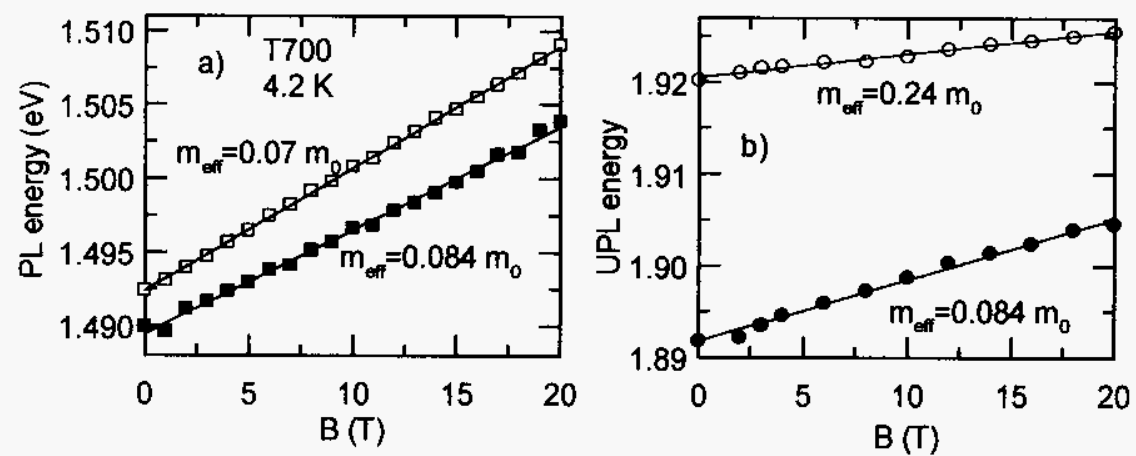

Figure 3. PL peak energies versus magnetic field $B$ in the T700 sample for a) the two peaks around $1.49 \mathrm{eV}$ and $\mathrm{b}$ ) the two structures observed in the UPL spectrum of GaInP. The values of the effective masses $\left(m_{e f f}\right)$ deduced from their slopes are indicated.

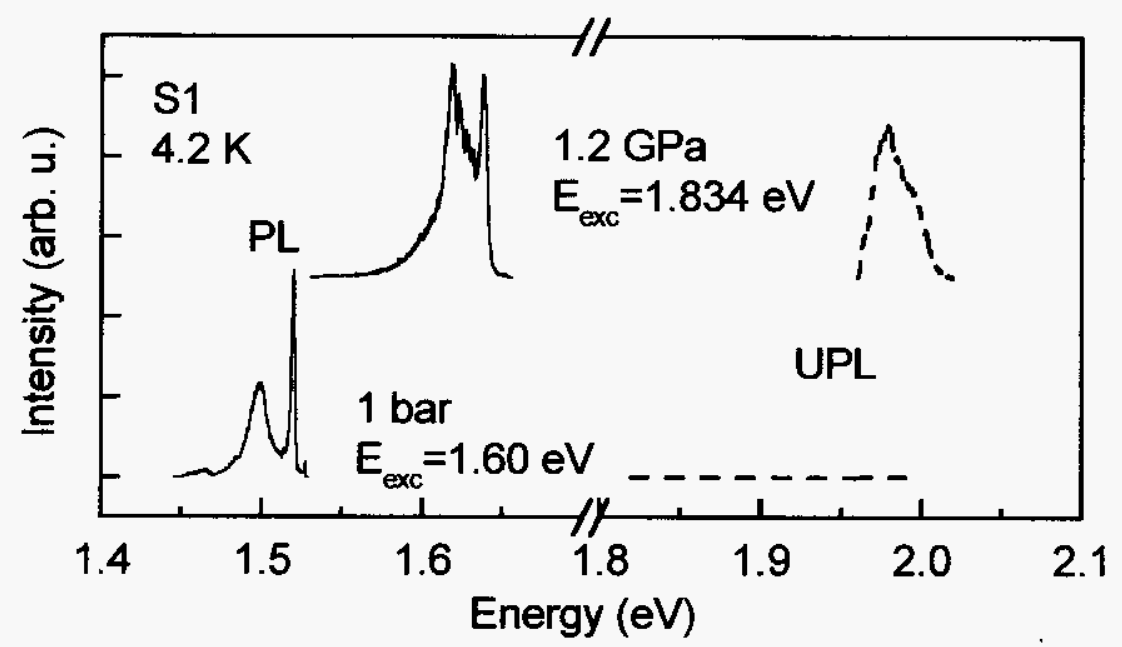

Figure 4. PL (continuous curves) and UPL (broken curves) spectra of the sample S1 measured at 1 bar and at $1.2 \mathrm{GPa}$. 